\title{
Supernova Acceleration Probe: Studying Dark Energy with Type Ia Supernovae
}

\author{
A White Paper to the Dark Energy Task Force
}

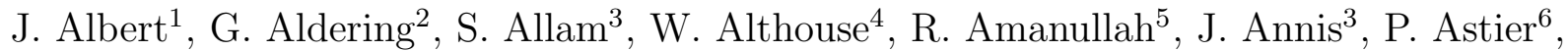
M. Aumeunier ${ }^{7,8}$, S. Bailey ${ }^{2}$, C. Baltay ${ }^{9}$, E. Barrelet ${ }^{6}$, S. Basa ${ }^{8}$, C. Bebek ${ }^{2}$, L. Bergström ${ }^{5}$, G. Bernstein ${ }^{10}$, M. Bester ${ }^{11}$, B. Besuner ${ }^{11}$, B. Bigelow ${ }^{12}$, R. Blandford ${ }^{4}$, R. Bohlin ${ }^{12}$,

A. Bonissent ${ }^{7}$, C. Bower ${ }^{13}$, M. Brown ${ }^{12}$, M. Campbell ${ }^{12}$, W. Carithers ${ }^{2}$, D. Cole ${ }^{14}$,

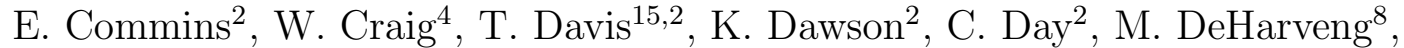

F. DeJongh ${ }^{3}$, S. Deustua ${ }^{16}$, H. Diehl ${ }^{3}$, T. Dobson ${ }^{11}$, S. Dodelson ${ }^{3}$, A. Ealet ${ }^{7,8}$, R. Ellis ${ }^{1}$,

W. Emmet ${ }^{9}$, D. Figer ${ }^{12}$, D. Fouchez ${ }^{7}$, M. Frerking ${ }^{14}$, J. Frieman ${ }^{3}$, A. Fruchter ${ }^{12}$,

D. Gerdes ${ }^{12}$, L. Gladney ${ }^{10}$, G. Goldhaber ${ }^{11}$, A. Goobar ${ }^{5}$, D. Groom ${ }^{2}$, H. Heetderks ${ }^{11}$,

M. Hoff ${ }^{2}$, S. Holland ${ }^{2}$, M. Huffer ${ }^{4}$, L. Hui ${ }^{3}$, D. Huterer ${ }^{17}$, B. Jain ${ }^{10}$, P. Jelinsky ${ }^{11}$,

C. Juramy ${ }^{6}$, A. Karcher ${ }^{2}$, S. Kent ${ }^{3}$, S. Kahn ${ }^{4}$, A. Kim², W. Kolbe², B. Krieger ${ }^{2}$,

G. Kushner ${ }^{2}$, N. Kuznetsova ${ }^{2}$, R. Lafever ${ }^{2}$, J. Lamoureux ${ }^{2}$, M. Lampton ${ }^{11}$, O. Le Fèvre ${ }^{8}$,

V. Lebrun ${ }^{8}$, M. Levi ${ }^{2}$, P. Limon ${ }^{3}$, H. Lin ${ }^{3}$, E. Linder ${ }^{2}$, S. Loken ${ }^{2}$, W. Lorenzon ${ }^{12}$,

R. Malina ${ }^{8}$, L. Marian ${ }^{10}$, J. Marriner ${ }^{3}$, P. Marshall ${ }^{4}$, R. Massey ${ }^{18}$, A. Mazure ${ }^{8}$,

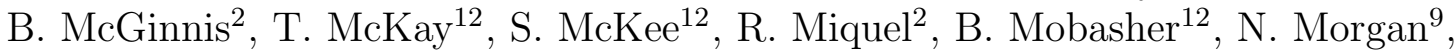

E. Mörtsell ${ }^{5}$, N. Mostek ${ }^{13}$, S. Mufson ${ }^{13}$, J. Musser ${ }^{13}$, R. Nakajima ${ }^{10}$, P. Nugent ${ }^{2}$,

H. Oluṣeyi ${ }^{2}$, R. Pain ${ }^{6}$, N. Palaio ${ }^{2}$, D. Pankow ${ }^{11}$, J. Peoples ${ }^{3}$, S. Perlmutter ${ }^{\dagger}$, D. Peterson ${ }^{2}$,

E. Prieto ${ }^{8}$, D. Rabinowitz ${ }^{9}$, A. Refregier ${ }^{19}$, J. Rhodes ${ }^{1,14}$, N. Roe ${ }^{2}$, D. Rusin $^{10}$,

V. Scarpine ${ }^{3}$, M. Schubnell ${ }^{12}$, M. Seiffert ${ }^{14}$, M. Sholl ${ }^{11}$, H. Shukla ${ }^{11}$, G. Smadja ${ }^{20}$,

R. M. Smith ${ }^{1}$, G. Smoot ${ }^{11}$, J. Snyder ${ }^{9}$, A. Spadafora ${ }^{2}$, F. Stabenau ${ }^{10}$, A. Stebbins ${ }^{3}$,

C. Stoughton ${ }^{3}$, A. Szymkowiak ${ }^{9}$, G. Tarlé12, K. Taylor ${ }^{1}$, A. Tilquin ${ }^{7}$, A. Tomasch ${ }^{12}$,

D. Tucker ${ }^{3}$, D. Vincent ${ }^{6}$, H. von der Lippe ${ }^{2}$, J-P. Walder ${ }^{2}$, G. Wang ${ }^{2}$, A. Weinstein ${ }^{1}$,

W. Wester ${ }^{3}$, M. White ${ }^{11}$

saul@lbl.gov

melevi@lbl.gov

${ }^{1}$ California Institute of Technology

${ }^{2}$ Lawrence Berkeley National Laboratory

${ }^{3}$ Fermi National Accelerator Laboratory

${ }^{4}$ Stanford Linear Accelerator Center

${ }^{5}$ University of Stockholm

${ }^{6}$ LPNHE, CNRS-IN2P3, Paris, France

${ }^{7}$ CPPM, CNRS-IN2P3, Marseille, France

${ }^{8}$ LAM, CNRS-INSU, Marseille, France

${ }^{9}$ Yale University

${ }^{10}$ University of Pennsylvania

${ }^{11}$ University of California at Berkeley

${ }^{12}$ University of Michigan

${ }^{12}$ Space Telescope Science Institute

${ }^{13}$ Indiana University

${ }^{14}$ Jet Propulsion Laboratory

${ }^{15}$ The Australian National University

\section{Executive Summary}

The Supernova Acceleration Probe (SNAP) will use Type Ia supernovae (SNe Ia) as distance indicators to measure the effect of dark energy on the expansion history of the Universe. (SNAP's weak-lensing program is described in a separate White Paper.) The experiment exploits super-

\footnotetext{
${ }^{16}$ American Astronomical Society

${ }^{17}$ University of Chicago

${ }^{18}$ Cambridge University

${ }^{19}$ CEA, Saclay, France

${ }^{20}$ IPNL, CNRS-IN2P3, Villeurbanne, France

${ }^{*} \mathrm{Co}-\mathrm{PI}$

${ }^{\dagger} \mathrm{PI}$
} 
nova distance measurements up to their fundamental systematic limit; strict requirements on the monitoring of each supernova's properties leads to the need for a space-based mission. Results from pre-SNAP experiments, which characterize fundamental SN Ia properties, will be used to optimize the SNAP observing strategy to yield data, which minimize both systematic and statistical uncertainties. With early R\&D funding, we have achieved technological readiness and the collaboration is poised to begin construction. Pre-JDEM AO R\&D support will further reduce technical and cost risk. Specific details on the SNAP mission can be found in Aldering et al. (2004, 2005).

\subsection{Overview of Goals and Techniques}

The primary goal of the SNAP supernova program is to provide a dataset which gives tight constraints on parameters which characterize the dark-energy, e.g. $w_{0}$ and $w_{a}$ where $w(a)=w_{0}+$ $w_{a}(1-a)$. SNAP data can also be used to directly test and discriminate among specific darkenergy models. We will do so by building the Hubble diagram of high-redshift supernovae, the same methodology used in the original discovery of the acceleration of the expansion of the Universe that established the existence of dark energy (Perlmutter et al. 1998; Garnavich et al. 1998; Riess et al. 1998; Perlmutter et al. 1999).

The SNAP SN Ia program focuses on minimizing the systematic floor of the supernova method through the use of characterized supernovae that can be sorted into subsets based on subtle signatures of heterogeneity. Subsets may be defined based on host-galaxy morphology, spectral-feature strength and velocity, early-time behavior, inter alia. Independent cosmological analysis of each subset of "like" supernovae can be compared to give confidence that the results are free from significant systematics. Conversely, analysis between supernova subsets at the same redshift can identify further systematics controls. While theories of the supernova progenitor and explosion mechanism can guide the establishment of subset criteria, such understanding is not required - only comprehensive measurements are - for robustness of the cosmological results. The level of robustness is tied to the quality of data with which supernovae are distinguished. Statistical mission requirements are fundamentally bound by the systematic limi- tations of the experiment.

\subsection{Description of the Baseline Proposal}

SNAP is a 2-m space telescope (shown in Figure 1) with a 0.7 square-degree instrumented focal plane imager (shown in Figure 2) and a low resolution $R \sim 100$ integral-field-unit spectrograph (schematic shown in Figure 3). Both instruments are sensitive to the $0.4-1.7 \mu \mathrm{m}$ wavelength range. The two SNAP deep-survey fields each subtends 7.5 square degrees and each is observed approximately every four days over the time-scales of supernova light-curve evolution in each of nine passbands. The passbands have resolution $\sim 4.5$ and are logarithmically distributed over the full wavelength range. Targeted spectrographic observations are made of discovered supernovae with exposure times tuned to the source flux.

The magnitude depths for individual scans and co-added images of the SNAP supernova fields are calculated for each filter. Table 1 shows the limiting $S / N=5 \mathrm{AB}$ magnitude for each filter in the "Deep" supernova survey, for point sources that are not contaminated with cosmic rays. The limiting magnitude for any given point is probabilistic, due to the random occurrence of cosmic rays. In a single scan of the deep survey, $77 \%$ of point sources will have no cosmic-ray contamination in any of the four dithered images while $98 \%$ will have one or fewer cosmic-contaminated image, only slightly reducing signal-to-noise.

\section{Precursor Observations}

Any precursor mission that characterizes the fundamental properties of SNe Ia and their applicability as distance indicators is important for optimizing the observing strategy and data analysis of SNAP.

Low-redshift surveys provide high signal-tonoise, comprehensive observations of well-characterized SNe Ia and could provide specific requirements for the observation of light-curve or spectroscopic features linked to SN Ia heterogeneity and homogeneity. Heterogeneous features provide a handle to probe the systematic limitations of our distance indicator and form the criteria by which "like" supernova subsets are defined. Conversely, current distance measurements to supernovae may be found not to be optimal; a different mix of ob- 
TABLE 1

The SNAP AB Magnitude SURVEy DePth For a POINT source $S / N=5 .^{\text {a }}$

\begin{tabular}{ccccccc}
\hline \hline \multirow{2}{*}{ Filter } & $\lambda_{\text {eff }}(\AA)$ & $\Delta \lambda(\AA)$ & \multicolumn{2}{c}{ Deep Survey (AB mag) } & \multirow{2}{*}{$\begin{array}{c}\text { Wide-field Survey } \\
\text { (AB mag) }\end{array}$} & $\begin{array}{c}\text { Panoramic } \\
\text { (ABmag) }\end{array}$ \\
\cline { 4 - 5 } & & & Per Scan & Co-added Scans & & \\
\hline 1 & 4400 & 1000 & 27.9 & 30.6 & 28.3 & 26.7 \\
2 & 5060 & 1150 & 27.9 & 30.6 & 28.3 & 26.8 \\
3 & 5819 & 1322 & 27.8 & 30.5 & 28.2 & 26.8 \\
4 & 6691 & 1520 & 27.8 & 30.4 & 28.1 & 26.8 \\
5 & 7695 & 1749 & 27.8 & 30.4 & 28.1 & 26.8 \\
6 & 8849 & 2011 & 27.7 & 30.3 & 28.0 & 26.7 \\
7 & 10177 & 2313 & 27.5 & 30.1 & 27.8 & 26.6 \\
8 & 11704 & 2660 & 27.5 & 30.1 & 27.8 & 26.6 \\
9 & 13459 & 3059 & 27.4 & 30.0 & 27.7 & 26.5 \\
\hline
\end{tabular}

${ }^{a}$ Random cosmic-ray hits make the $S / N$ for a given position probabilistic; see text. The choice of filter set is currently subject to optimization studies; the filters and depths presented here are meant to be illustrative.

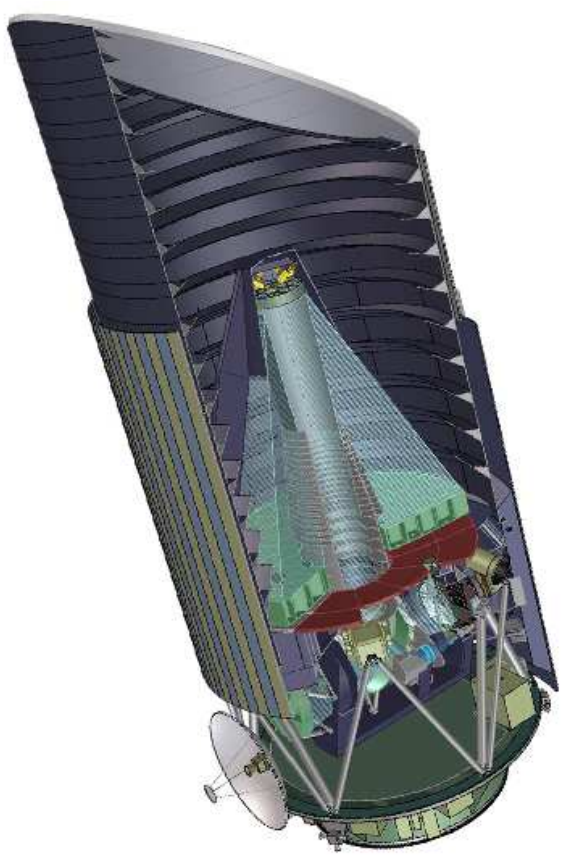

Fig. 1.- Cutaway view of the reference SNAP design. servations may accentuate supernova homogeneity and increase the probative power of a single event. Finally, these surveys will provide an expanded library of spectra for K-corrections.

Ongoing high-redshift supernova searches and SNAP probe similar depths and employ similar observing strategies. Precursor missions establish high-redshift supernova rates and fluxes which will impact the SNAP survey strategy. The search and follow-up of "rolling" supernova searches (continuous repeated observing of the target fields) provide a testbed for SNAP discovery and trigger for spectroscopic follow-up. These searches may indicate that specific observables are linked to redshift-dependent evolution in supernova populations. Finally, these searches can access and allow the study of the supernova-frame UV which has unexploited potential for aiding in supernova cosmology measurements.

\section{Error budget}

SNe Ia have already proved to be excellent distance indicators for probing the dynamics of the Universe. In order to move toward the era of precision cosmology, a new data set must both address potential systematic uncertainties and provide sufficient statistical power to accurately determine 


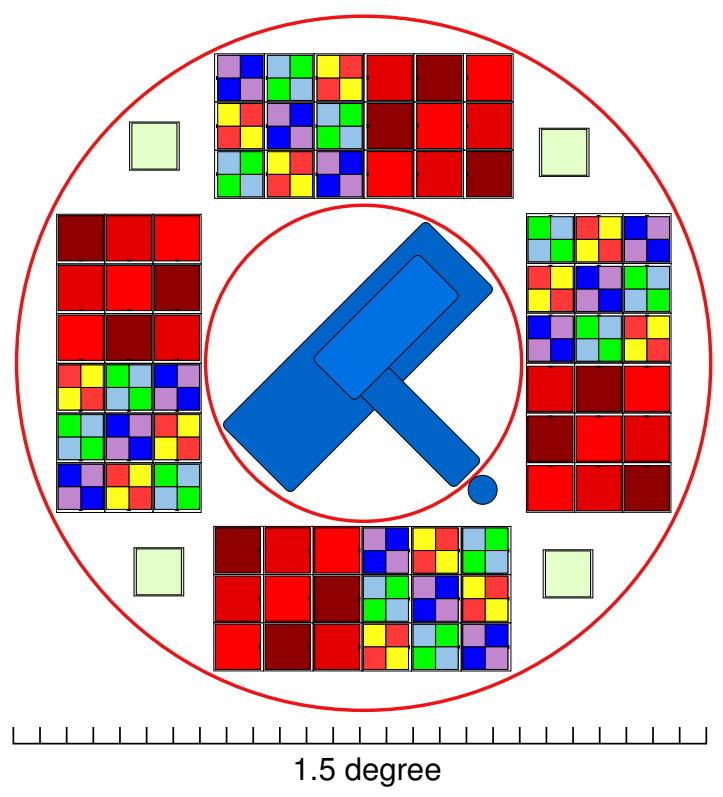

Fig. 2.- The SNAP focal plane working concept. The two-axis symmetry of the imager filters allows any $90^{\circ}$ rotation to scan a fixed strip of the sky and measure all objects in all nine filter types. The imager covers 0.7 square degrees. Underlying the filters, there are $362 \mathrm{k} \mathrm{x} 2 \mathrm{k} \mathrm{HgCdTe} \mathrm{NIR} \mathrm{devices} \mathrm{and}$ $363.5 \mathrm{k} \times 3.5 \mathrm{k}$ CCDs on a $140 \mathrm{~K}$ passively-cooled focal plane. The six CCD filter types and three NIR filters are arranged so that vertical or horizontal scans of the array through an observation field will measure all objects in all filters. The false colors indicate filters with the same bandpass. The central rectangle and solid circle are the spectrograph body and its light access port, respectively. The spectrum of a supernova is taken by placing the star in the spectrograph port by steering the satellite. The four small, isolated squares are the star guider CCDs. The inner and outer radii are 129 and $284 \mathrm{~mm}$, respectively.

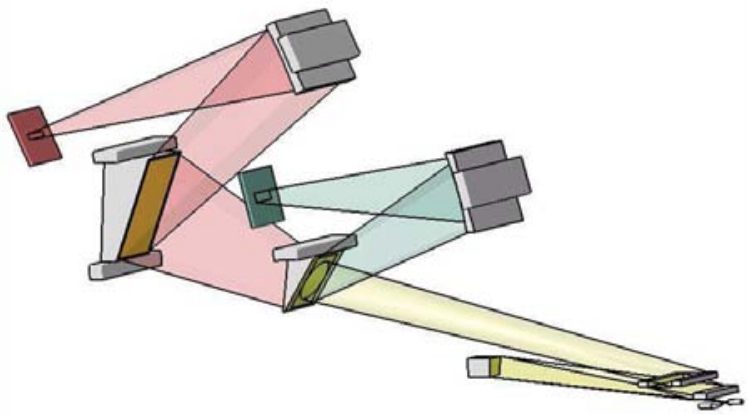

Fig. 3.- A schematic spectrograph optical design. The beam is going out from the slicer (on the bottom right) to a prism disperser back faced coated by a dichroic. The visible light (blue path) is reflected and the IR light (in red) continue to a second prism used to reach the required spectral dispersion. The two beams are therefore focused on two detectors. The dimensions of the spectrograph are approximately $400 \times 80 \times 100 \mathrm{~mm}$. See Ealet et al. (2003) for more details.

dark-energy properties, especially the time variation of the equation of state.

\subsection{Sources of Systematic Uncertainty}

High-redshift supernova searches have been proceeding since the late 1980's (Norgaard-Nielsen et al. 1989; Couch et al. 1989; Perlmutter et al. 1995, 1997, 1998, 1999; Schmidt et al. 1998; Riess et al. 1998; Tonry et al. 2003; Knop et al. 2003; Riess et al. 2004). Particularly since the discovery of the accelerating expansion of the Universe, the high-redshift supernova methodology for measuring cosmological parameters has been critically scrutinized for sources of systematic uncertainty. Below (and summarized in Table 2) is a lists of these sources where we highlight in bold the features specific to SNAP that allow systematic control. Generically, the need for high signal-to-noise observations over a broad wavelength range for $0.1<z<1.7$ supernovae, as well as the truncation of ground-observatory light curves of high-redshift supernova at high-Galactic latitude, point to the necessity of a space mission. 
TABLE 2

SNAP Control of Sources of Astrophysical Systematic UnCertainty

\begin{tabular}{|c|c|}
\hline Systematic & Control \\
\hline Supernova evolution & $\begin{array}{l}\text { Use of only the best-characterized supernovae subclassified through high } \\
\text { signal-to-noise light curves and spectrum(a) }\end{array}$ \\
\hline K Correction & $\begin{array}{l}\text { Tuning of the SNAP passbands and accumulation of a library of supernova } \\
\text { spectra }\end{array}$ \\
\hline Galactic extinction & Search fields in the direction of the low-extinction Galactic caps \\
\hline Cross-passband calibration & A SNAP program to construct a set of high-precision flux standards \\
\hline Host-galaxy dust & $\begin{array}{l}\text { Wavelength-dependent absorption identified with high signal-to-noise } \\
\text { multi-band photometry spanning a broad wavelength range }\end{array}$ \\
\hline Gravitational lensing & $\begin{array}{l}\text { Measure the average flux for a large number of supernovae in each redshift } \\
\text { bin }\end{array}$ \\
\hline Non-Type Ia contamination & Classification of each event through the $6150 \AA$ SiII and S spectral features \\
\hline Malmquist bias & Supernovae discovered early with high signal-to-noise photometry \\
\hline Gray dust & $\begin{array}{l}\text { Photometry and spectroscopy for supernovae at wavelengths where gray } \\
\text { dust is no longer achromatic to search for gray dust at moderate redshift }\end{array}$ \\
\hline
\end{tabular}

\subsubsection{Evolution of the Supernova Sample as a Function of Redshift}

The luminosity function of supernovae may evolve as a function of redshift resulting in corresponding biases in distance-modulus determinations (Perlmutter et al. 1995). Although like supernovae have the same redshift-independent luminosity, their relative rate of occurrence may evolve in redshift.

SNAP addresses this problem by identifying subsets of supernovae "identical" in luminosity and color, applying the dark-energy analysis to each, and optimally combining the subset results into final cosmology measurements. We exploit the fact that supernovae cannot change their brightness in one measured wavelength range without affecting brightness somewhere else in the spectral time series - an effect that is wellcaptured by expanding atmosphere computer models (e.g. Höflich \& Khokhlov 1996; Lentz et al. 2000; Höflich et al. 2003). Well-characterized supernovae can be sorted into subsets based on subtle signatures of heterogeneity. Subsets may be defined based on host-galaxy morphology, spectralfeature strength and velocity, early-time behavior, and light-curve characteristics.

There are a number of supernova parameters that are observed to exhibit heterogeneity beyond the single-parameter description. The following features need to be monitored to check for systematic bias in supernova distances.

Premaximum spectral screening: Broad-lightcurve supernovae can be distinguished by having either peculiar (e.g. SN 1991T) or "normal" (e.g. SNe 1999ee and 2000E) pre-maximum spectra. In addition, there are extremely peculiar singleton events technically classified as SNe Ia which bear little observational resemblance to their more standard counterparts. These include SN 2000cx (Li et al. 2001; Candia et al. 2003), SN 2001ay (Phillips et al. 2003), SN 2002cx (Li et al. 2003), and SN 2002ic (Deng et al. 2004). These extreme events are easily identified through their premaximum peculiar spectra, Hydrogen emission, and high expansion velocities.

Spectral-feature velocities: The diversity in the velocity of the $6100 \AA$ SiII feature at fixed epochs has been noted (Branch \& van den Bergh 1993; Hatano et al. 2000). Garavini et al. (2004) and Benetti et al. (2004) have examined the velocity as a function of supernova phase and find that large subset of objects tightly cluster within a $10^{3}$ $\mathrm{km} \mathrm{s}^{-1}$ range for any given epoch. A smaller subset with much higher velocity dispersion can be partially distinguished through accurate measurements of the Sill feature velocity. 
Spectral-feature velocity evolution: Benetti et al. (2004) find that supernovae cluster when parameterized by the rate of change of the SiII feature velocity $\dot{v}$ and luminosity. Resolution of the velocity gradients requires measurement of $\dot{v}$ to a few $\mathrm{km} \mathrm{s}^{-1} \mathrm{~d}^{-1}$ from spectra between peak and 40 SN-frame days after peak brightness.

Spectral-line-ratio: At $t<-10$, the ratio of line depths of $\operatorname{SiII}(\lambda 6150 \AA)$ and $\operatorname{SiII}(\lambda 5800 \AA), \mathrm{R}(\mathrm{SiII})$ (Nugent et al. 1995), has a range greater than 0.1 . The subpopulation of supernovae with high velocity gradients also have distinct $\mathrm{R}(\mathrm{SiII})$ evolution; they sharply decrease until $t=-5$ and flatten out whereas other supernovae show no evolution for the same time range (Benetti et al. 2004).

Light-curve evolution: There are a series of supernovae that exhibit systematic deviations in light-curve shape and color (e.g. Hamuy et al. 1996; Richmond et al. 1995; Krisciunas et al. 2003; Pignata et al. 2004). The ranges displayed in the optical light curves can be summarized as follows: $1 \mathrm{mag} 10$ days before maximum and $0.2 \mathrm{mag} 5$ days before maximum, 0.1 mag 20-30 days after maximum, and 0.2-0.3 mag 60 days after maximum.

Color: There is evidence that color is a second parameter correlated with peak magnitude (Tripp 1998; Saha et al. 1999; Tripp \& Branch 1999; Parodi et al. 2000). Dust extinction and intrinsic supernova color can be distinguished as supernovae get brighter in $I$ as $V-I$ color becomes red, the opposite effect from dust. Color measurements whose propagated uncertainties in the extinction correction are less than the intrinsic $B$ peak magnitude dispersion correspond to $B-V$ measured to $0.07 \mathrm{mag}$ and $V-I$ to $0.5 \mathrm{mag}$.

Polarization: Normal- and broad-light-curve supernovae have demonstrated varying degrees of polarization (Howell et al. 2001). Detection of polarization is extremely difficult for all but the nearest supernovae and is unrealistic to require in any cosmological survey. Spectroscopy may then serve as a proxy for polarization measurements through their correlation with high and quickly evolving SiII velocities.

The SNAP instrumentation suite is designed to provide the data necessary for the subclassification process. SNAP will obtain broadband photometry over the full supernova-frame optical region, with fine light-curve sampling tuned to natural supernova time scales. The SNAP spectrograph also covers the supernova-frame optical region with resolution tuned to the widths of the supernova features.

We expect that any residual luminosity and color biases will be smaller than the dispersion observed in the local supernova sample since this represents heterogeneous and diverse supernovaprogenitor environments (Branch 2001). This assumption will be tested by intensive precursor supernova-characterization experiments and SNAP itself.

\subsubsection{K-correction}

A given observing passband is sensitive to different supernova-rest-frame spectral regions. Kcorrections are used to put these differing photometry measurements onto a consistent rest-frame passband (Kim, Goobar, \& Perlmutter 1996; Nugent, Kim, \& Perlmutter 2002). The SNAP filter-set number, density, and shapes are tuned to give $<0.02 \mathrm{mag}$ dispersion for the full catalog of currently available SN Ia spectra (Davis et al. 2004). The situation will improve further for the confined analysis of supernova subsamples with the expanded spectral library provided by ongoing surveys and SNAP itself.

\subsubsection{Galactic Extinction}

Extinction maps of our own Galaxy are uncertain by $\sim 1-10 \%$ depending on direction (Schlegel, Finkbeiner, \& Davis 1998). Supernova fields can be chosen toward the low-extinction Galactic caps where uncertainty can then be controlled to $<$ $0.5 \%$ in brightness. These levels of uncertainty have negligible effect on the measurement of $w_{0}$ and $w_{a}$ (Kim \& Miquel 2005). Spitzer observations will allow an improved mapping between color excesses (e.g. of Galactic halo subdwarfs in the SNAP field) and Galactic extinction by dust (Lockman \& Condon 2005).

\subsubsection{Cross-Passband Calibration}

Uncertainties in calibration are one of the important limiting factors in probing the dark energy with supernovae. Distance measurements fundamentally rely on the comparison of fluxes in dif- 
ferent passbands: for determining the relative distances of supernovae at different redshifts and for color measurements for determining the dust absorption of a single supernova.

The relationship between passband calibration and $w_{0}$ and $w_{a}$ is strongly dependent on the specifics of the cross-band correlations. Simulation studies (Kim \& Miquel 2005) demonstrate the strict fundamental limits in the measurement of dark-energy parameters due to uncorrelated crossband calibration uncertainty. Significantly larger correlated uncertainties can be tolerated (Kim et al. 2004). These studies provide requirements for the SNAP calibration program.

\subsubsection{Extinction by Host-Galaxy Dust}

Extinction from host-galaxy dust can significantly reduce the observed brightness of a discovered supernova. SNAP's 9-band photometry will identify the properties of the obscuring dust and gas, and thus the amount of extinction suffered by individual supernovae. Incoherent scatter in dust properties yields no bias but increased statistical uncertainty in the dark-energy parameters (Linder \& Miquel 2004). Systematic host-galaxy extinction uncertainty can be introduced if an incorrect mean dustextinction law is used in the analysis. This can be minimized by distinguishing and excluding highly-extincted supernovae from the analysis. To give a quantitative example, suppose that the Cardelli et al. (1989) model accurately describes host-galaxy extinction and an $R_{V}=3.1$ dust is used in the analysis and the $4400-5900 \AA$ rest-frame color is used to determine $A_{V}$. We calculate that if drift in the mean of the true dust extinction models is constrained to have $2.4<R_{V}<4.4$, the vast majority of supernova which have $A_{B}<0.1$ (Hatano, Branch, \& Deaton 1998 ) would have distance-modulus bias of $<0.02$ mag.

\subsubsection{Gravitational Lensing}

Inhomogeneities along the supernova line of sight can gravitationally magnify or demagnify the supernova flux and shift the mode of the supernova magnitude distribution by $\lesssim 3 \%$ depending on redshift (Holz \& Linder 2005). Gravitational lensing magnification is achromatic and unbiased in flux so effectively contributes a statistical uncertainty that can be reduced below $1 \%$ in distance if large numbers $(\gtrsim 70)$ of supernovae are obtained per 0.1 redshift bin. The effective flux dispersion induced by lensing goes as $\sigma=0.088 z$ (Holz \& Linder 2005).

\subsubsection{Non-SN Ia Contamination}

Other supernova types are on average fainter than SNe Ia and their contamination could bias their Hubble diagram (Perlmutter et al. 1999). Observed supernovae must be positively identified as SN Ia. As some Type Ib and Ic supernovae have spectra and brightnesses that otherwise mimic those of SNe Ia (Filippenko 1997), a spectrum covering the defining rest frame Si II 6150Å feature and SII features for every supernova at maximum will provide a pure sample.

\subsubsection{Malmquist Bias}

A flux-limited sample preferentially detects the intrinsically brighter members of any population of sources. The amount of magnitude bias depends on details of the search but can reach the level of the intrinsic magnitude dispersion of a standard candle (Teerikorpi 1997). Directly correcting this bias would rely on knowledge of the SN Ia luminosity function, which may change with lookback time. A detection threshold fainter than peak by at least five times the intrinsic SN Ia luminosity dispersion ensures sample completeness with respect to intrinsic supernova brightness, eliminating this bias (Kim et al. 2004).

\subsubsection{Extinction by Hypothetical Gray Dust}

A systematics-limited experiment must account for speculative but reasonable sources of uncertainty such as gray dust. As opposed to normal dust, gray dust is postulated to produce wavelength-independent absorption in optical bands (Aguirre 1999; Aguirre \& Haiman 2000). Although models for gray-dust grains dim blue and red optical light equally, near-infrared light $(\sim 1-2 \mu \mathrm{m})$ is less affected. Cross-wavelength calibrated spectra extending to wavelength regions where "gray" dust is no longer gray will detect and correct for the hypothetical 
large-grain dust. Numerous observations already severely limit the cosmological effects of such a component (Goobar, Bergström, \& Mörtsell 2002).

\subsection{Statistical Supernova Sample}

The statistical supernova sample appropriate for experiments with the presence of systematic uncertainty has been considered in the Fishermatrix analysis of Linder \& Huterer (2003) and validated with our independent Monte Carlo analysis. They examined the measurement precision of dark-energy parameters as a function of redshift depth considering 2000 SNe Ia measured in the range $0.1 \leq z \leq z_{\max }$, along with 300 lowredshift SNe Ia from the Nearby Supernova Factory (Aldering et al. 2002). They concluded that a SN Ia sample extending to redshifts of $z \sim 1.7$ is crucial for realistic experiments in which some systematic uncertainties remain after all statistical corrections are applied. Ignoring systematic uncertainties can lead to claims that are too optimistic.

Statistically, $\sim 2000$ SNe Ia well-characterized "Branch-normal" supernovae provide systematicuncertainty limited measurements of the darkenergy parameter $w_{a}$. These represent the cream, $z<1.7$ supernovae of the $\sim 10000$ total and $\sim 4000$ well-characterized SNAP SNe Ia, that can provide robust cosmological measurements (e.g. non-peculiar, low-extinction). This large sample is necessary to allow model-independent checks for any residual systematics or refined standardization parameters, since the sample will be subdivided in a multidimensional parameter space of redshift, light curve-width, host properties, etc. The large number of lower signal-to-noise light curves from supernovae at $z>1.7$ will provide an extended redshift bin in which to check for consistency with the core sample.

We require a statistical measurement uncertainty for the peak magnitude corrected for extinction and shape inhomogeneity roughly equal to the current intrinsic magnitude dispersion of supernovae, $\sim 0.15 \mathrm{mag}$.

\section{Quantification of Dark Energy}

Expected measurement precisions of the darkenergy parameters $w_{0}$ and $w^{\prime}\left(=w_{a} / 2\right)$ for the
SNAP supernova program are summarized in Table 3 and shown in Figure 4. Since SNAP is a systematics-limited experiment, any improvements in our projection of systematic uncertainty will be directly reflected in improvements in our $w_{0}-w^{\prime}$ measurements. We determine precisions for two fiducial flat universes with $\Omega_{M}=0.3$, one in which the dark energy is attributed to a cosmological constant and the other to a SUGRA-inspired dark-energy model (Brax \& Martin 2000).

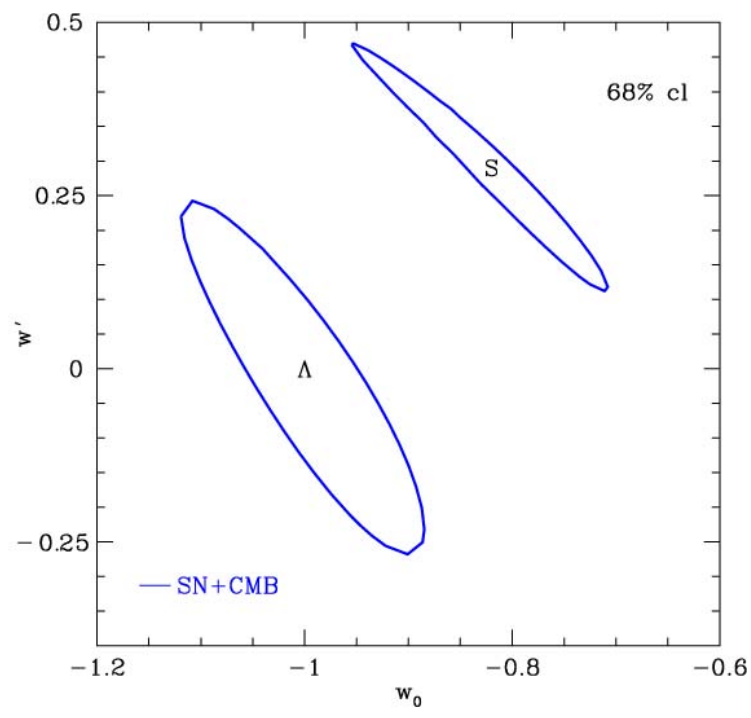

Fig. 4.- The $68 \%$ confidence region in the darkenergy parameters for fiducial cosmological constant (labeled with $\Lambda$ ) and supergravity (labeled with S) universes. The blue solid curves show the results of the supernova simulation and associated systematic-error model, with a Planck prior on the distance to the surface of last scattering, and an assumed flat universe. We define $w^{\prime} \equiv-d w /\left.d \ln a\right|_{z=1}$. See Albert et al. (2005) for constraints including the weak lensing half of the mission.

If the universe is taken to be spatially flat, the SN data (with Planck prior on the distance to the surface of last scattering) determine $\Omega_{M}$ to 0.01 . The present value of the dark energy equation of state is constrained within 0.09 and the physically crucial dynamical clue of the equation of state time variation is bounded to within 0.19. Note that these constraints are systematics limited; much tighter constraints would be obtained if systematics were ignored. Table 3 also high- 
TABLE 3

SNAP 1- $\sigma$ UNCERTAINTIES IN DARK-ENERGY PARAMETERS, WITH CONSERVATIVE SYSTEMATICS FOR THE SUPERNOVA AND THE SHEAR POWER-SPECTRUM MEASUREMENT FROM A 1000 SQ. DEG. ONE-YEAR WeAK-Lensing survey. Note: These uncertainties are systematics-Limited, not statistics LIMITED.

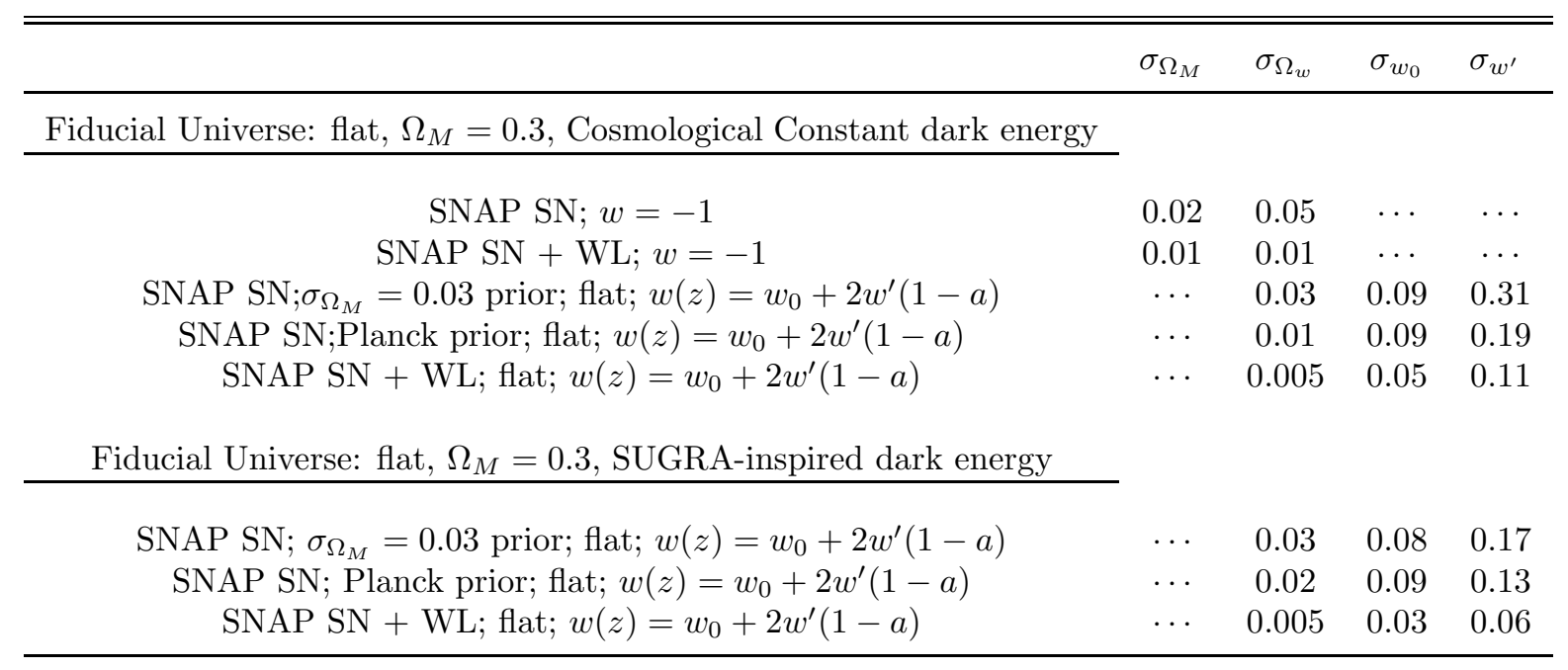

Note. - Cosmological and dark-energy parameter precisions for two fiducial flat universes with $\Omega_{M}=$ 0.3, one in which the dark energy is attributed to a Cosmological Constant and the other to a SUGRAinspired dark-energy model. The parameter precisions then depend on the choice of data set, priors from other experiments (e.g. Planck measurement of the distance to the surface of last scattering), assumptions on the flatness of the universe, and the model for the behavior of $w$. SNAP is a systematicslimited experiment so any improvements in our projection of systematic uncertainty will be directly reflected in improvements in our $w_{0}-w^{\prime}$ measurements. In this paper we define $w^{\prime} \equiv-d w /\left.d \ln a\right|_{z=1}$. SNAP can accumulate a wide-field weak-lensing survey at a rate of 1000 sq. deg. per year of observation. 
lights the significant value added by measurements of the shear power spectrum with the complementary SNAP weak-lensing program.

The precise, homogeneous, deep supernova data set can alternatively be analyzed in a non-parametric, uncorrelated bin, or eigenmode method (Huterer \& Starkman 2003; Huterer \& Cooray 2005). One can also obtain the expansion history $a(t)$ itself, e.g. Linder (2003).

\section{Project Risks and Strengths}

The proposed experiment is a self-contained supernova program which is desensitized to crossexperimental calibration, one of the largest sources of systematic uncertainty. The SNAP instrumentation suite provides access to any supernova observable anticipated to be important for reducing uncertainties in supernova distances.

The SNAP collaboration is large and diverse, which has allowed for substantial efforts to be supported in science reach, computer framework and simulation, calibration approaches, and photodetector technology development and testing. Our ongoing R\&D efforts have placed us at a point of technological readiness to commence construction of the experiment.

The major risk to the SNAP collaboration is that a stretch in the JDEM decision making process and follow-on project funding will lead to a dissipation of the present efforts as people drift off to other intermediate projects.

\section{Requisite technology R\&D}

A few areas of the SNAP mission implementation require development to achieve flight readiness. No new technologies are required, rather particular instances of the technology as chosen by SNAP require some maturation. At present, the SNAP R\&D program is primarily funded by the Department of Energy (DOE), with additional support to collaborators from NSF, NASA, CNES, and CNRS/IN2P3 for related activities. The SNAP mission architecture has undergone numerous studies (GSFC/IMDC, GSFC/ISAL, and JPL/Team-X), has been reviewed several times by external teams of experts at the request of DOE and NSF, and has been extensively presented and discussed at a variety of international conferences.
Thus, SNAP is a relatively well-advanced concept, and the internally self-consistent reference model presented here is now ready for more refined optimization.

R\&D has focused on 1) SNAP-specific detector development, both visible and near infrared, 2) integral field unit spectrograph design, 3) cold frontend electronics, 4) calibration procedures and supporting flight hardware, 5) achievable telescope point stability studies, 6) on-board data handling, and 7) achievable telemetry rate studies. All of these areas are maturing at a rapid rate and there appear to be no show stoppers for a SNAP JDEM response.

Visible detectors: The visible portion of the imager is an array of 36 silicon CCDs with extended red response and improved radiation tolerance. SNAP will materially benefit from an advanced technology based on fully depleted backside illuminated high resistivity silicon. The development work is DOE funded and is taking place at LBNL and at commercial foundries. We are also developing custom integrated circuits for the electronic readout.

NIR detectors: The NIR portion of the imager requires $362 \mathrm{kx} 2 \mathrm{k}$ format devices. We are evaluating $\mathrm{HgCdTe}$ devices available from two vendors. Development work funded by DOE is under way to improve the dark current, read noise, and quantum efficiency. We are also exploring InGaAs as a backup photoactive material. One NIR vendor will provide a cold front-end ASIC for their detectors. For the other, we are leveraging the work we have done for the CCDs to provide cold front-end ASICs.

Spectrograph: This is a simple two-prism design with no technology challenges except its integral field unit (IFU). An image-slicer IFU with the requisite size and efficiency have been built for ground-based astronomy, and will be flown as part of the JWST NIRSPEC. The image slicer R\&D is funded by CNES and CNRS/IN2P3.

Telescope: This has been studied by the Goddard/ISAL and four prospective space optics suppliers. Two industry study contracts are in place to evaluate the design, manufacturability, and testability of the telescope. Studies and vendor discussions have shown that the pointing stability requirements can be met with today's spacecraft 
technology.

Data handling: We have developed an on-board detector data handling concept where each detector operation, data processing, and storage is done in parallel. This allows for graceful degradation of the focal plane performance at the detector level. The large integrated data storage required and the way it is used favors FLASH RAM technology. Initial radiation testing indicates that this is a viable approach.

Telemetry: We require a $150 \mathrm{Mbit} / \mathrm{s}$ Ka-band downlink. While the ground portion of this system can be bought "off the shelf", and heritage Kaband TWTA transmitters of the required power are readily available, the $150 \mathrm{MHz}$ modulator is a development item. Signal processing upgrades will be required to existing ground stations to handle $150 \mathrm{Mbit} / \mathrm{s}$.

Calibration: An important feature of the supernova-cosmology analysis is its dependence on the relative brightness of Type Ia supernovae. The SNAP calibration program must thus precisely control the absolute color calibration in the $0.4-1.7 \mu \mathrm{m}$ observing window. We plan to establish a set of astronomical sources with known physical fluxes as "fundamental standards". A network of fainter primary and secondary standard stars, accessible to SNAP and larger ground-based telescopes, are to be established with respect to the fundamental standards. The requirements on the accuracy of the calibration standards are defined by their propagated effect on $w_{0}$ and $w_{a}$.

\section{Facility Access}

To receive the SNAP science data, access to the NASA Deep Space Network or other NASA ground stations is desired. Upgrades to process $150 \mathrm{Mbit} / \mathrm{s}$ demodulation are required.

\section{Timeline}

With the considerable detector and mission development which has already been completed, the SNAP collaboration is ready to begin construction of the experiment.

\section{REFERENCES}

Aguirre, A. 1999, ApJ, 512, L19

Aguirre, A. \& Haiman, Z. 2000, ApJ, 532, 28
Albert, J. et al. 2005, ArXiv Astrophysics eprints,astro-ph/05074xxx

Aldering, G. et al. 2002, Proc. SPIE, 4836, 61

-. 2004, astro-ph, 0405232

Aldering, G. et al. 2005, in Wide-Field Imaging From Space, ed. A. Fruchter, E. V. Linder, \& T. McKay, astro-ph/0507426

Benetti, S. et al. 2004, MNRAS, 348, 261

Branch, D. 2001, Bulletin of the American Astronomical Society, 33, 1481

Branch, D. \& van den Bergh, S. 1993, AJ, 105, 2231

Brax, P. \& Martin, J. 2000, Phys. Rev., D61, 103502

Candia, P. et al. 2003, PASP, 115, 277

Cardelli, J. A., Clayton, G. C., \& Mathis, J. S. 1989, ApJ, 345, 245

Couch, W. J. et al. 1989, in Particle Astrophysics: Forefront Experimental Issues (Singapore; Teaneck, NJ: World Scientific), 192

Davis, T., Schmidt, B. P., Kim, A., \& SNAP. 2004, Bulletin of the American Astronomical Society, 205 ,

Deng, J. et al. 2004, ApJ, 605, L37

Ealet, A. et al. 2003, Proc. SPIE, 4850

Filippenko, A. V. 1997, ARA\&A, 35, 309

Garavini, G. et al. 2004, AJ, 128, 387

Garnavich, P. M. et al. 1998, ApJ, 509, 74

Goobar, A., Bergström, L., \& Mörtsell, E. 2002, A\&A, 384, 1

Hamuy, M. et al. 1996, AJ, 112, 2438

Hatano, K., Branch, D., \& Deaton, J. 1998, ApJ, 502,177

Hatano, K., Branch, D., Lentz, E. J., Baron, E., Filippenko, A. V., \& Garnavich, P. M. 2000, ApJ, 543, L49 
Höflich, P., Gerardy, C., Linder, E., \& Marion, H. 2003, in Stellar candles for the extragalactic distance scale, ed. D. Alloin \& W. Gieren, Lecture notes in physics,0075-8450 ;635 (Berlin; New York: Springer)

Höflich, P. \& Khokhlov, A. 1996, ApJ, 457, 500

Holz, D. E. \& Linder, E. V. 2005, ApJ, accepted

Howell, D. A., Höflich, P., Wang, L., \& Wheeler, J. C. 2001, ApJ, 556, 302

Huterer, D. \& Cooray, A. 2005, Phys. Rev. D, 71, 023506

Huterer, D. \& Starkman, G. 2003, Phys. Rev. Lett., 90, 031301

Kim, A., Goobar, A., \& Perlmutter, S. 1996, PASP, 108, 190

Kim, A. \& Miquel, R. 2005, In preparation

Kim, A. G., Linder, E. V., Miquel, R., \& Mostek, N. 2004, MNRAS, 347, 909

Knop, R. A. et al. 2003, ApJ, 598, 102

Krisciunas, K. et al. 2003, AJ, 125, 166

Lentz, E. J., Baron, E., Branch, D., Hauschildt, P. H., \& Nugent, P. E. 2000, ApJ, 530, 966

Li, W. et al. 2001, PASP, 113, 1178

—. 2003, PASP, 115, 453

Linder, E. V. 2003, Phys. Rev. Lett., 90, 91301

Linder, E. V. \& Huterer, D. 2003, Phys. Rev. D, 67,81303

Linder, E. V. \& Miquel, R. 2004, Phys. Rev. D, 70,123516

Lockman, F. J. \& Condon, J. J. 2005, AJ, 129, 1968

Norgaard-Nielsen, H. U., Hansen, L., Jorgensen, H. E., Aragon Salamanca, A., \& Ellis, R. S. 1989, Nature, 339, 523

Nugent, P., Kim, A., \& Perlmutter, S. 2002, PASP, 114, 803

Nugent, P., Phillips, M., Baron, E., Branch, D., \& Hauschildt, P. 1995, ApJ, 455, L147
Parodi, B. R., Saha, A., Sandage, A., \& Tammann, G. A. 2000, ApJ, 540, 634

Perlmutter, S. et al. 1995, ApJ, 440, L41

—. 1997, ApJ, 483, 565

-. 1998, Nature, 391, 51

-. 1999, ApJ, 517, 565

Phillips, M. M. et al. 2003, in From Twilight to Highlight: The Physics of Supernovae, 193

Pignata, G. et al. 2004, MNRAS, 433

Richmond, M. W. et al. 1995, AJ, 109, 2121

Riess, A. G. et al. 1998, AJ, 116, 1009

—. 2004, ApJ, 607, 665

Saha, A., Sandage, A., Tammann, G. A., Labhardt, L., Macchetto, F. D., \& Panagia, N. 1999, ApJ, 522, 802

Schlegel, D. J., Finkbeiner, D. P., \& Davis, M. 1998, ApJ, 500, 525

Schmidt, B. P. et al. 1998, ApJ, 507, 46

Teerikorpi, P. 1997, ARA\&A, 35, 101

Tonry, J. L. et al. 2003, ApJ, 594, 1

Tripp, R. 1998, A\&A, 331, 815

Tripp, R. \& Branch, D. 1999, ApJ, 525, 209

This 2-column preprint was prepared with the AAS LATEX macros v5.2. 